\title{
Norois
}

Environnement, aménagement, société

226 | 2013

Commerce et développement local, déplacements urbains, valorisations agricoles, découpages électoraux

\section{Les déplacements urbains et la pollution de l'air dans des villes intermédiaires : enjeux politiques et environnementaux}

Urban mobility and air pollution in intermediate cities: urban planning and environmental stakes

\section{Lise-Marie Glandus et Gérard Beltrando}

\section{OpenEdition} Journals

Édition électronique

URL : https://journals.openedition.org/norois/4538

DOI : 10.4000/norois.4538

ISBN : 978-2-7535-2287-9

ISSN : $1760-8546$

\section{Éditeur}

Presses universitaires de Rennes

\section{Édition imprimée}

Date de publication : 30 mars 2013

Pagination : 25-40

ISBN : 978-2-7535-2285-5

ISSN : 0029-182X

\section{Référence électronique}

Lise-Marie Glandus et Gérard Beltrando, « Les déplacements urbains et la pollution de l'air dans des villes intermédiaires : enjeux politiques et environnementaux», Norois [En ligne], 226 | 2013, mis en ligne le 30 mars 2015, consulté le 13 janvier 2022. URL : http://journals.openedition.org/norois/4538 DOI : https://doi.org/10.4000/norois.4538 


\title{
Les déplacements urbains et la pollution de l'air dans des villes intermédiaires : enjeux politiques et environnementaux
}

\author{
Urban Mobility and Air Pollution in Intermediate Cities: \\ Urban Planning and Environmental Stakes
}

\author{
Lise-Marie Glandus ${ }^{*}$, Gérard Beltrando ${ }^{\mathrm{b}}$
}

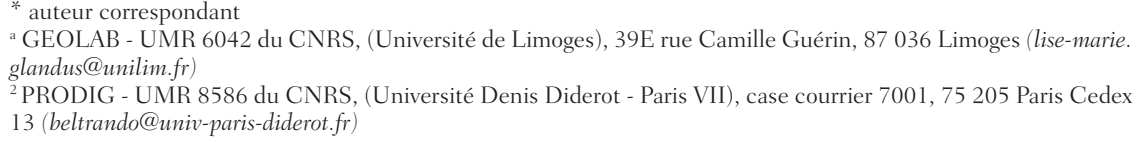

Résumé : Les déplacements quotidiens en voiture représentent aujourd'hui la principale cause de pollution atmosphérique en milieu urbain. Ce problème, particulièrement présent dans les très grandes villes, concerne également - mais dans une moindre mesure - les agglomérations intermédiaires, à l’image de La Rochelle, Limoges et Clermont-Ferrand. Celles-ci sont alors amenées à remettre en question l'organisation des espaces urbains, encore fortement centrée sur l'usage du véhicule particulier, par le biais des Plans de Déplacements Urbains. Au-delà de la législation nationale, l'analyse des actions locales révèle des inégalités dans la prise en compte de l'environnement et de l'aménagement urbain, notamment en termes d'évolution des réseaux de transports alternatifs à la voiture, mais aussi des écarts entre les projets et les réalisations concrètes. Des limites peuvent alors être mises en avant, qu'elles dépendent des échelles d'élaboration des politiques, des relations entre les différents acteurs, de l'intérêt accordé à la pollution de l'air ou des volontés d'implication.

Abstract: At the present time, regular car mobility is the main cause of atmospheric pollution in urban areas. This problem, particularly present in the biggest cities, also concerns - to a lesser extent - intermediate agglomerations, such as La Rochelle, Limoges and Clermont-Ferrand. These latter are induced to question urban planning, while automobile represents the central point of organization, through the "Plans de Déplacements Urbains". Beyond the national legislation, analysis of local actions shows differences in attention paid to environment and urban planning, particularly in terms of alternatives to driving evolution as well as gaps between plans and implementations. Bounds appear, linked to policies working out scales, relations between the different participants, interest showing in air pollution or wills of implication.

Mots clés : pollution atmosphérique - agglomérations intermédiaires - périurbanisation - mobilités quotidiennes - aménagements urbains - transports collectifs

Keywords: atmospheric pollution - intermediate agglomerations - peri-urbanization - regular mobility - urban planning - public transport 


\section{INTRODUCTION}

Le trafic automobile constitue aujourd'hui la principale source d'émissions en milieu urbain et les déplacements quotidiens sont à l'origine d'une pollution atmosphérique diversifiée (Kaufmann et al., 2001 ; CITEPA, 2011). La périurbanisation a entraîné une dispersion de l'habitat et des zones d'activités, et surtout un accroissement des flux de mobilité entre les espaces périphériques et les communes-centres. Ces modifications de l'organisation urbaine concernent aussi bien les très grandes villes, à l'image de Paris, que les agglomérations de taille intermédiaire (Datar, 2010), telles que La Rochelle, Limoges et Clermont-Ferrand. Ce sont ces dernières qui ont été choisies ici, en raison de leur moindre développement spatial et démographique que celui des grandes villes. Ce caractère suppose, en effet, une pollution bien plus faible et, en conséquence, moins de préoccupations et d'investissements politiques dans le domaine environnemental. Les enjeux de la pollution de l'air s'y trouvent d'ailleurs moins médiatisés que dans les grandes villes et les principaux bassins industriels où les problèmes sont beaucoup plus marqués. Pourtant, ces trois villes sont soumises à une même législation, commune aux agglomérations de plus de 100000 habitants. Dans un contexte urbain marqué par l'étalement spatial et la place centrale de l'automobile, la loi sur l'air de 1996 et la loi Solidarité et Renouvellement Urbains de 2000, notamment, se sont orientées vers la prise en compte commune des questions de pollution et d'aménagement urbain (Drobenko, 2002). Les agglomérations possèdent alors de nombreuses responsabilités dans ce domaine. Mais on peut s'interroger sur la réelle prise en considération des enjeux de pollution et la pertinence des politiques de déplacements et d'aménagement au sein de ces villes intermédiaires, dans un contexte fortement marqué par les mobilités quotidiennes et la place prépondérante du réchauffement climatique dans l'actualité.

\section{Des agglomérations INTERMÉDIAIRES MARQUÉES PAR LA POLLUTION DE L'Air}

La pollution de l'air met en jeu de nombreuses substances, dont les effets ne doivent pas être négligés, à l'origine d'une contamination de petite échelle (la planète) comme de grande échelle (l'es- pace local). En milieu urbain, la pollution émane principalement de trois sources : les activités industrielles et les activités relatives au secteur domestique (chauffage, incinération des ordures ménagères), historiquement les plus anciennes, ainsi que le secteur des déplacements automobiles, désormais considéré comme la source d'émissions la plus notable au sein des agglomérations (CITEPA, 2011 ; Dab et Roussel, 2001 ; Masclet, 2005).

\section{Une pollution atmosphérique fortement dépendante de l'évolution urbaine}

Les villes intermédiaires tendent à être considérées, a priori, comme des espaces à la qualité de vie agréable, où la pollution atmosphérique ne constitue pas un problème. Ce point de vue est d'autant plus valable à La Rochelle, Limoges et Clermont-Ferrand, que celles-ci appartiennent pour les deux premières villes à des régions modérément industrialisées et présentent un développement démographique et économique moindre que celui des grandes villes (respectivement 185000, 260000 et 427000 habitants au sein des aires urbaines en 2006). Toutefois, ces trois sites sont fortement marqués par le phénomène de périurbanisation, développé dès les années 1960, qui a considérablement modifié l'organisation originelle des espaces urbains. La multiplication des pôles périphériques, qu’ils soient de nature résidentielle, industrielle ou commerciale, implique l'existence de flux de mobilité quotidiens importants. Les aires urbaines ${ }^{1}$ constituent alors la meilleure représentation des agglomérations fonctionnelles, car correspondant à un "système spatial socioéconomique fait de mouvements et dont on évalue les dimensions à partir des fonctions productives : navettes domicile-travail, secteurs d'activité, niveaux de services » (Lévy et Lussault, 2003).

Cet étalement urbain a ainsi conduit à donner au véhicule particulier un rôle central dans l'organisation de ces agglomérations. En conséquence, les habitants de La Rochelle, Limoges et ClermontFerrand présentent un recours majoritaire à l'automobile pour les usages quotidiens, domicile-travail en particulier, comme le montrent les résultats

1. Pour l'INSEE, une aire urbaine est « un ensemble de communes, d'un seul tenant et sans enclave, constitué par un pôle urbain, et par des communes rurales ou unités urbaines (couronne périurbaine) dont au moins $40 \%$ de la population résidente ayant un emploi travaillent dans le pôle ou dans des communes attirées par celui-ci ». 
du recensement de l'INSEE relatifs aux modes de déplacements (tableau 1).

\begin{tabular}{|l|c|c|c|c|}
\hline & Voiture & Marche & Deux roues & $\begin{array}{c}\text { Transports } \\
\text { en commun }\end{array}$ \\
\hline La Rochelle & 78,2 & 6,1 & 7,0 & 4,7 \\
\hline Limoges & 80,2 & 8,1 & 2,1 & 6,1 \\
\hline Clermont-Ferrand & 76,2 & 9,2 & 3,1 & 7,9 \\
\hline Lyon & 68,1 & 7,9 & 3,3 & 17,2 \\
\hline Paris & 43,7 & 7,4 & 4,3 & 40,8 \\
\hline France métropolitaine & 69,7 & 7,7 & 4,1 & 14,2 \\
\hline
\end{tabular}

Tableau 1 : Modes de déplacements pour les trajets domicile-travail au sein des aires urbaines et comparaisons françaises (Source : INSEE, RP 2008)

Transport modes for journeys to work in functional urban regions and French comparisons

Les transports en commun, qui constituent le mode le plus apte à concurrencer la voiture au sein des aires urbaines, à la fois pour des trajets de courte et longue distances, se trouvent particulièrement délaissés. Limoges présente cependant une plus grande utilisation automobile, alors que les habitants de Clermont-Ferrand montrent un plus faible recours au véhicule particulier et un usage plus marqué des transports en commun.

Ces différences conduisent donc à s'interroger sur leurs origines et, notamment, sur le degré d'implication des agglomérations en termes d'aménagements urbains locaux et de politiques des transports.

\section{Les centres-villes, plus vulnérables aux concentrations polluantes}

L'étude des niveaux moyens de pollution au sein de ces trois agglomérations révèle la forte place du trafic automobile dans la pollution locale, même si les mesures réalisées par les Associations Agréées de Surveillance de la Qualité de l'Air (AASQA) dépendent également des facteurs climatiques et topographiques, qui influent sur la concentration ou la dispersion des polluants, ainsi que des émissions issues de certaines installations industrielles.

Les données prises en compte ici proviennent de stations de mesures de natures différentes (trafic, urbaines et périurbaines de fond ${ }^{2}$ ), sur la période

2. Les stations de type trafic sont situées à moins de $5 \mathrm{~m}$ des voies de circulation et mesurent une pollution directement influencée par les émissions routières, à laquelle sont soumis les riverains, les automobilistes et les piétons. Les stations de fond (urbaines et périurbaines) sont localisées dans des espaces relativement éloignés des sources d'émissions et présentent donc la qualité de l'air moyen respiré en zone urbaine (dans les du $1^{\text {er }}$ janvier 1999 au 28 décembre 2006, pour les concentrations moyennes journalières en quatre polluants : dioxyde d'azote $\left(\mathrm{NO}_{2}\right)$, dioxyde de soufre $\left(\mathrm{SO}_{2}\right)$, particules en suspension de diamètre inférieur à 10 microns (PM10) et ozone $\left(\mathrm{O}_{3}\right)$. Ceux-ci correspondent aux sous-indices de l'indice ATMO destiné à informer la population sur les niveaux de pollution quotidiens et sont ainsi les plus couramment surveillés par les AASQA. En outre, le monoxyde de carbone $(\mathrm{CO})$, particulièrement présent dans les centres urbains et bon marqueur de la pollution d'origine automobile, a également été pris en considération lors de campagnes de mesures itinérantes réalisées au sein de l'hypercentre de Limoges, les dimanche 10 et lundi 11 février 2008 (Glandus et al., 2011 ; Dahech, 2007). À cette occasion, des mesures fixes d'une durée d'1 minute ont été effectuées sur onze points d'arrêt (fig. 1).

L'ensemble de ces mesures met en évidence les particularités des centres-villes et, notamment, leur exposition notable à la proximité du trafic automobile. Ces espaces sont marqués par des caractères particuliers, tels que des vitesses de circulation réduites, des ralentissements et redémarrages répétés, ainsi que des aménagements urbains influençant la concentration des polluants : des rues plutôt étroites bordées de bâtiments assez hauts, de larges boulevards, des places et des carrefours. Les premières sont reconnues comme étant plutôt propices à la concentration de la pollution quand elles ne sont pas orientées dans la direction du vent dominant, les substances polluantes ayant tendance à s'accumuler au niveau des voies de circulation (Xiaomin et al., 2006). Toutes ces conditions génèrent une présence plus marquée de la pollution, notamment dans des conditions atmosphériques stables.

En particulier, l'impact de la hausse du trafic automobile se manifeste de façon assez nette le long des rues et des places les plus fréquentées, en présence de vents de faible vitesse (moyenne de $1,5 \mathrm{~m} / \mathrm{s}$, soit $5,4 \mathrm{~km} / \mathrm{h}$ ). La pollution s'accroît sur ces voies (avenues, carrefours), mais aussi lors des arrêts des véhicules aux feux rouges. A l'occasion du circuit de mesures réalisé le lundi entre $16 \mathrm{~h} 40$ et $17 \mathrm{~h} 40$, les teneurs en CO augmentent à partir de la place Denis Dussoubs (point d'arrêt $\mathrm{n}^{\circ} 7$ sur la figure 1) et les valeurs sont particuliè-

centres urbains), périurbaine (à la périphérie des espaces centraux) ou rurale (dans des secteurs à faible densité de population). Dans ce cas, l'air ambiant est prélevé à environ $3 \mathrm{~m}$ au-dessus du substrat. 


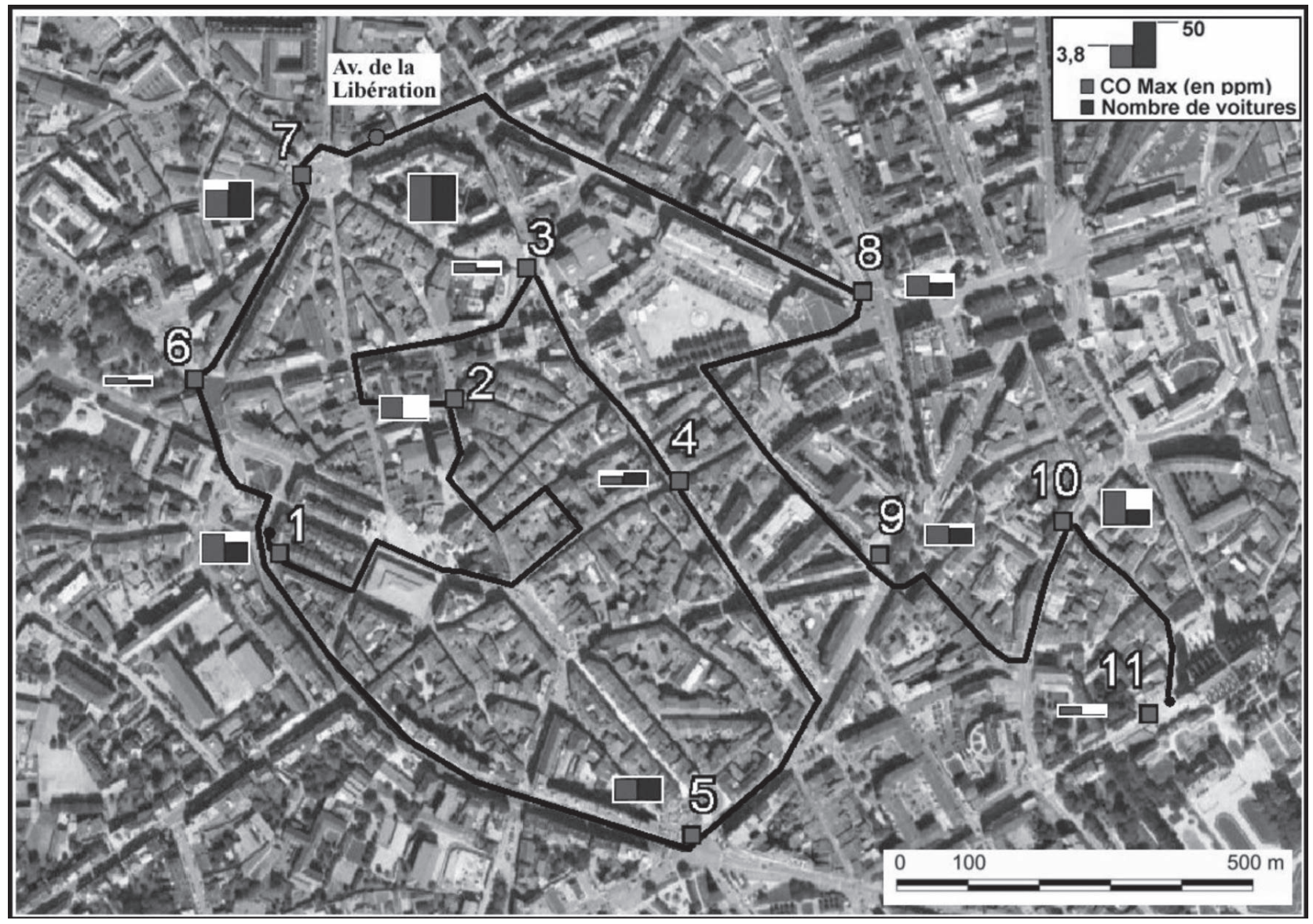

Figure 1 : Concentrations maximales en $\mathrm{CO}$ et voitures en circulation durant 1 min sur divers points d'arrêt dans le centre de Limoges, le lundi 11 février 2008 entre $16 \mathrm{~h} 40$ et $17 \mathrm{~h} 40$

(Fond de carte : Google Earth (C) 2009)

Maximum CO concentrations and car traffic during 1 mn at various stops in the Limoges town center on Monday 11th February 2008 between 4:40 and 5:40 pm

rement élevées dans l'avenue de la Libération (jusqu’à 7,6 ppm, soit $8816 \mu \mathrm{g} / \mathrm{m}^{3}$, correspondant au maximum enregistré lors de cette campagne).

De plus, certaines rues étroites, bien qu'étant peu fréquentées par les automobilistes, révèlent une pollution en CO plus marquée que celle d'artères larges et plus empruntées, en lien avec le passage de véhicules roulant à faible vitesse (moins de $30 \mathrm{~km} / \mathrm{h}$ ) et les dimensions des voies, assez peu favorables à la dispersion des polluants.

Enfin, les fluctuations hebdomadaires de pollution apparaissent de façon assez nette au sein des stations de type trafic, révélant la forte influence de la circulation automobile. De façon générale, les concentrations les plus élevées en $\mathrm{NO}_{2}, \mathrm{SO}_{2}$ et PM10 se manifestent pendant la semaine de travail, c'est-à-dire du lundi au vendredi (et notamment les mardi et mercredi), alors que la pollution diminue durant le week-end (figure 2). Ceci permet alors d'identifier le trafic automobile comme la principale source d'émissions, en lien avec l'organisation des activités urbaines (Lonati et al., 2006) : celles-ci étant plus intenses durant les jours de travail, le trafic automobile augmente et entraîne des hausses d'émissions qui sont visibles dans les mesures des stations.

Les problèmes sont surtout marqués sur les stations de type trafic, situées à proximité immédiate des voies de circulation. Les concentrations moyennes en $\mathrm{NO}_{2}$ et PM10 s'y trouvent souvent supérieures à l'objectif de qualité et à la valeur limite pour la santé humaine ${ }^{3}$, tout particulièrement à

3. L'objectif de qualité représente « un niveau de concentration de substances polluantes dans l'atmosphère, fixé sur la base des connaissances 
Figure 2 : Profils hebdomadaires moyens de NO2, SO2 et PM10 pour les stations «trafic » de Clermont-Ferrand (CF) et La Rochelle (LR), de 1999 à 2006 (Sources : Atmo Auvergne, Atmo Poitou-Charentes)

Average weekly profiles of pollution in the "trafic" stations of Clermont-Ferrand $(C F)$ and La Rochelle (LR) from 1999 to 2006

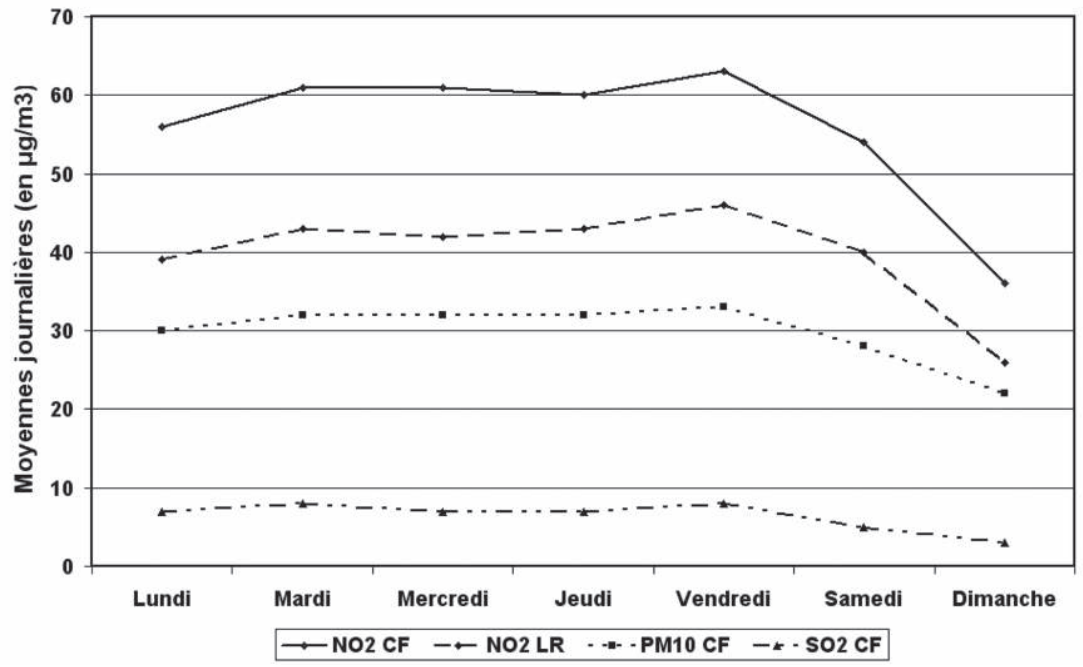

lement qu'une diminution de $25 \%$ des niveaux de pollution permettrait une baisse de $36 \%$ des décès anticipés et $42 \%$ des hospitalisations, alors que la pollution aurait été à l'origine de 28 décès anticipés en 1999-2000, 8 hospitalisations pour motif respiratoire, ainsi que 50 pour motif cardio-vasculaire en été et 24 en hiver (Cire, 2005).

Les principales conclusions issues de ces diverses études tendent à considérer qu'une simple réduction des dépassements des seuils réglementaires ne peut suffire à apporter un réel gain sanitaire. Une réduction des émissions à la source et sur le long terme constitue ainsi la meilleure solution, notamment en ce qui concerne la circulation automobile. Plus particulièrement, la forte variabilité spatiale et temporelle de la pollution qui caractérise les centres-villes, nécessiterait une prise en compte spécifique dans les aménagements urbains, dans le but de réduire la circulation automobile et les niveaux de pollution.

\section{DES OUTILS DE PLANIFICATION DES TRANSPORTS AU SERVICE DE LA QUALITÉ DE L'AIR}

En France, la naissance d'un débat relatif à la pollution automobile ne date que des années 1980 et reste assez hésitant, compte tenu de la présence d'un puissant lobbying des constructeurs. De ce fait, les avantages de la voiture ont plus été mis en avant que ses inconvénients en termes environnemental et sanitaire. Les parlementaires ont surtout cherché à soutenir et encourager le développement de l'indus-

scientifiques, dans le but d'éviter, de prévenir ou de réduire les effets nocifs de ces substances pour la santé humaine ou pour l'environnement »; la valeur limite pour la santé humaine représente " un niveau maximal de concentration de substances polluantes dans l'atmosphère, fixé sur la base des connaissances scientifiques, dans le but d'éviter, de prévenir ou de réduire les effets nocifs de ces substances pour la santé humaine ou pour l'environnement » (Loi n 96-1236 du 30 décembre 1996 : LAURE). 
trie automobile, celle-ci étant avant tout considérée comme une avancée économique et technologique (Vlassopoulou, 1999). La prise en considération de la pollution atmosphérique a donc été assez tardive, puisque ce n'est qu'en 1996 qu'une loi cadre sur l'air est votée et que l'État organise la surveillance et l'amélioration de la qualité de l'air. Différents échelons administratifs du pays sont concernés, du ministère de l'Écologie, du Développement Durable, des Transports et du Logement, jusqu'aux associations locales de surveillance et aux groupements intercommunaux, chargés de relier environnement et aménagement urbain en optimisant les modes de déplacement.

\section{Une conciliation entre environnement et aménagement urbain}

Dans ce contexte urbain accordant autant de place aux mobilités quotidiennes et aux modes de transport motorisés individuels, quelques réponses ont été apportées dans le but de réduire les impacts environnementaux du trafic automobile. Dans le cadre juridique, la principale avancée a été la Loi sur l'Air et l'Utilisation Rationnelle de l'Énergie (LAURE), instituée le 30 décembre 1996. Celle-ci transpose en droit français les normes européennes de la qualité de l'air et rend obligatoire, à l'échelle locale, la mise en place de la surveillance de la pollution de l'air, via les AASQA, et l'instauration d'outils de planification, dont les Plans de Déplacements Urbains (PDU).

Les PDU, mis en avant par la Loi d'Orientation des Transports Intérieurs (LOTI) en 1982, doivent désormais obligatoirement être instaurés sur l'espace des Périmètres de Transports Urbains (PTU) des agglomérations de plus de 100000 habitants. Les communes-centres, accompagnées de certaines de leurs communes périphériques, ont donc pour mission de gérer l'organisation de leur espace urbain, par le biais d'actions traitant à la fois le trafic et le stationnement, dans le sens d'un « usage coordonné de tous les modes de déplacements, notamment par une affectation appropriée de la voirie, ainsi que la promotion des modes les moins polluants et les moins consommateurs d'énergie » (loi n 96-1236 du 30 décembre 1996).

Ainsi, le développement des réseaux de transports publics, accompagné de mesures complémentaires, telles que le réaménagement de la voirie et du stationnement, ainsi que le développement des modes doux (marche et vélo), constituent les principaux outils disponibles en vue de diminuer la place de la voiture et entraîner une limitation des émissions polluantes.

Mais l'organisation urbaine actuelle, marquée par l'étalement spatial et la multiplicité des pôles d'attraction, incite à intégrer la gestion environnementale dans l'aménagement urbain. La loi relative à la Solidarité et au Renouvellement Urbain (SRU) du 13 décembre 2000 intègre justement les transports à la planification urbaine, avec pour objectif de « reconquérir le champ urbain, en optimisant ses perspectives d'évolution dans le périmètre urbanisé ». Il paraît en effet utile de mieux contrôler la périurbanisation diffuse par le biais de plusieurs opérations : le renforcement des pôles existants, la densification de l'urbanisation autour des axes de transports urbains, la diversification des fonctions urbaines dans le but de limiter le nombre et la longueur des déplacements, la promotion des modes doux et, de ce fait, le développement des commerces et services de proximité. Toutes ces orientations rejoignent finalement le modèle de la ville durable et compacte, prônant une densité et une mixité des fonctions urbaines, selon les principes énoncés dans la Charte d'Aalborg de 1994 et par opposition à ceux issus de la Charte d'Athènes de $1933^{4}$.

Une ville compacte associe densité et continuité urbaine, ainsi que mixité des fonctions, avec les avantages supposés en termes de mobilité, dans un contexte de réduction des distances de déplacement favorisant le recours aux modes doux et, notamment, la pratique de la marche à pied (Newman et Kenworthy, 1989; Dupuy, 1995; Newman et Kenworthy, 1999; Burton, 2000; Wiel, 2002), ainsi que des transports collectifs plus efficaces (Emangard, 1994; Kenworthy et Laube, 1999; Pouyanne, 2004) et par conséquent de moindres émissions polluantes (Frank et al., 2006).

Il reste malgré tout une certaine indécision générale, l'efficacité des formes urbaines compactes n'étant pas encore totalement démontrée et com-

\footnotetext{
4. Le principal concept issu de la Charte d'Athènes réside dans le constitution de zones indépendantes dédiées à chacune des quatre principales «fonctions » que sont : la vie, le travail, les loisirs et les transports. La Charte d'Aalborg ambitionne au contraire de sortir de ce cloisonnement qui a été particulièrement prisé dans le développement urbain depuis les années 1960.
} 
munément admise. La densification urbaine possède des limites d'ordre social, du fait de la réticence d'une grande part des habitants vis-à-vis d'un nouveau mode de vie : "La densité n'est pas synonyme de qualité de vie urbaine » (Paquot, 2009). La notion d'« intensité urbaine » est alors parfois préférée à celle de densité. L'intensité urbaine permet d'aller au-delà de la simple volonté matérielle d'organisation, en cherchant à prendre en compte l'expérience et les aspirations des habitants, dans le but de proposer une ville agréable à vivre et socialement équitable (Da Cunha et Kaiser, 2009). Elle vise la mise en œuvre d'une ville alliant mixités, proximité, accessibilité et qualité de vie, à toutes les échelles spatiales (Zunino, 2009). De plus, si la densité urbaine est susceptible de promouvoir le recours aux modes de transports alternatifs à la voiture et peut ainsi permettre une diminution des sources mobiles de pollution, elle contribue à la concentration des populations dans les quartiers centraux et péricentraux au sein desquels la qualité de l'air est la moins bonne, en dépit d'une baisse des émissions dues au trafic automobile (Marshall et al., 2005; De Ridder et al., 2008; Bartholomew et Ewing, 2009; Schweitzer et Zhou, 2010). Linhalation des polluants pourrait également être renforcée dans les villes compactes du fait d'un accroissement des déplacements (marche à pied et vélo) les plus exposés à la pollution (de Nazelle et al., 2009; Hixson et al., 2010; Schweitzer et Zhou, 2010).

Les politiques d'aménagement urbain pourraient donc tenir compte de ces facteurs d'ordre sanitaire, en évitant ce qui serait qualifié de « remplissage », et prendre en considération l'impact du bâti et de la morphologie urbaine dans son ensemble sur la concentration de la pollution : la densité d'urbanisation se révèle en effet néfaste à la dispersion des polluants, ceci étant renforcé par l'influence des constructions sur l'écoulement du vent et l'existence des îlots de chaleur urbain. Les solutions sont alors susceptibles de passer par une extension des surfaces végétalisées ou encore une adaptation architecturale (Boutefeu, 2007 ; Colombert, 2008; Leroux, 2008).

Enfin, le modèle de ville durable étant perfectible, la réorganisation urbaine n'est pas totalement en mesure de résoudre le problème des mobilités et peut s'accompagner d'un réel développement des modes de transports alternatifs à la voiture.

\section{Des Plans de Déplacements Urbains très orientés vers le développement des transports en commun}

Les actions futures des aménageurs pourraient placer les transports alternatifs à la voiture au centre des plans de circulation et de construction. Les PDU des agglomérations de La Rochelle (2000), Limoges (2003) et Clermont-Ferrand (2001) affichent des volontés communes, principalement axées sur :

- l'amélioration de l'offre en transports en commun et surtout en Transports en Commun en Site Propre (TCSP);

- la restriction de la circulation automobile en centre-ville;

- la gestion du stationnement;

- le partage de la voirie entre les modes (voiture, transports publics, modes doux);

- l'accroissement de l'intermodalité;

- la limitation du phénomène de périurbanisation.

Les transports en commun constituent la clé de voûte de ces projets. Quelle que soit leur forme (train, tramway, bus, trolleybus), ils représentent une véritable alternative au véhicule particulier, aussi bien pour des trajets de courtes distances que des déplacements plus longs, issus de la périurbanisation. À l'inverse, l'usage des modes doux (déplacements piétonniers et vélo - qui constituent des moyens totalement non polluants -) est exclusivement réservé aux déplacements de courte distance et se trouve également fortement dépendant d'autres facteurs, tels que la topographie locale et les conditions météorologiques.

L'extension des réseaux urbains de Transports en Commun en Site Propre (TCSP) apparaît alors comme une solution permettant d'augmenter le potentiel d'attraction des transports en commun, ce dispositif impliquant un partage de la voirie qui lui est favorable et une réduction des obstacles induits par la circulation automobile (Certu et ADEME, 2002). Cet aménagement permet en effet d'accroître la vitesse de circulation des véhicules et permet aussi de garantir une meilleure ponctualité et une plus grande régularité. À Clermont-Ferrand, l'un des principaux avantages du tramway (mis en service en octobre 2006) réside dans sa vitesse de circulation $(22 \mathrm{~km} / \mathrm{h})$, rendue possible par un site propre intégral et la priorité aux feux. En outre, 
une réduction de la voirie réservée aux automobilistes complique leur déplacement et peut s'avérer dissuasive quant à l'usage de la voiture. Mais cela nécessite qu'une nette amélioration des transports publics soit instaurée, particulièrement en termes de fréquence des passages. Dans le cas contraire, les agglomérations s'exposent à ce que les réductions de voirie entraînent de plus forts encombrements et, par conséquent, une hausse de la pollution.

Le développement des transports en commun paraît donc prioritaire, mais la promotion des modes alternatifs passe également par le développement des voies réservées aux modes doux et de l'intermodalité, la restriction du stationnement en centre-ville et des conditions de circulation des automobilistes. En définitive, l'ensemble de ces actions semblerait nécessaire pour obtenir un résultat probant, car l'une sans l'autre ne saurait être suffisamment capable de modifier les comportements actuels.

\section{Des POLITIQUES DE DÉPLACEMENTS AUX MULTIPLES LIMITES}

L'exposé des objectifs de chacun des Plans de Déplacements Urbains répond aux impératifs de la loi sur l'air et de la loi SRU. Mais la réalité révèle l'écart existant entre les ambitions affichées et la mise en œuvre des actions. Plusieurs faiblesses apparaissent dans la mise en application des orientations des PDU, comme dans l'élaboration même de ces documents.

\section{Des échelles d'élaboration incohérentes}

Tout d'abord, l'échelle de mise en application des PDU se distingue comme le principal point faible de ces outils. Les Périmètres de Transports Urbains (PTU), qui correspondent aux échelles d'élaboration des plans de déplacements, se confondent le plus souvent avec les communautés d'agglomération. Ces dernières, qui constituent des Établissement Public de Coopération Intercommunale (EPCI), sont les maîtres d'ouvrage des PDU. S'il semble légitime, et même indispensable, que les politiques d'aménagement et de transports soient menées à l'échelle des agglomérations, les groupements intercommunaux n'apparaissent pas comme les meilleurs garants d'une bonne planification. Le réel problème réside en réalité dans l'incohérence de leur organisation.
En effet, celle-ci répond avant tout à des choix politiques et ne repose pas sur la réalité des modes de vie et, surtout, des mobilités. De ce fait, les périmètres des communautés d'agglomération ne permettent pas de prendre en compte toutes les mobilités entre communes-centres et périphérie, qui concernent aujourd'hui la totalité des aires urbaines. Or, ce découpage, reconnu comme le plus cohérent face aux critères des emplois et des déplacements quotidiens, ne correspond pas aux choix administratifs et politiques des communautés d'agglomération (figure 3). Les communautés d'agglomération correspondent donc aux agglomérations « morphologiques » et non aux agglomérations « fonctionnelles » (Mainet et Edouard, 2009).

L'incohérence de la communauté d'agglomération de Limoges (Limoges Métropole) est la plus flagrante. Si, à La Rochelle et Clermont-Ferrand, la totalité des communes de première couronne est intégrée au sein des communautés d'agglomération, ce n'est pas le cas à Limoges, où l'une des sept communes (Couzeix : 7700 habitants en 2007) n'a pas souhaité adhérer à ce groupement intercommunal. D'autres communes limitrophes (Aixe-sur-Vienne et Verneuil-sur-Vienne : 5600 et 4000 habitants en 2007), avec lesquelles les échanges sont nombreux - une majorité de la population active de ces communes travaillant au sein de l'unité urbaine de Limoges - sont également exclues du PDU, et par conséquent, du réseau de transports en commun urbain.

En conséquence, les réseaux de transports en commun souffrent d'un manque d'unité. Ce problème transparaît notamment à Limoges où le réseau urbain ignore de nombreux espaces urbains pourtant denses, en lien avec la forte incohérence du territoire de la communauté d'agglomération et une absence de concertation entre groupements intercommunaux sur le thème des déplacements. Ce manque d'harmonisation conduit alors à une utilisation plus fréquente de la voiture particulière.

Ainsi, il apparaît que les choix politiques sont trop prééminents face aux logiques fonctionnelles de mobilité, alors que la gestion de la pollution de l'air et des politiques de transport ne peut pas être réduite à un espace trop restreint, qui découle d'une organisation administrative et politique. La mise en œuvre des PDU à l'échelle des communautés d'agglomération serait donc préjudiciable à l'efficacité 
Aire urbaine de La Rochelle

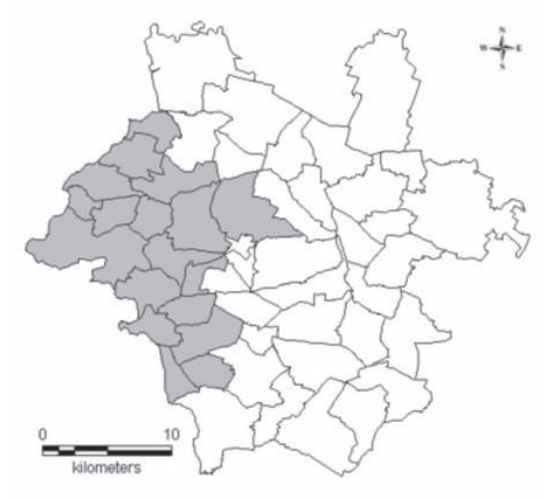

Communautés d'agglomération
Aire urbaine de Limoges

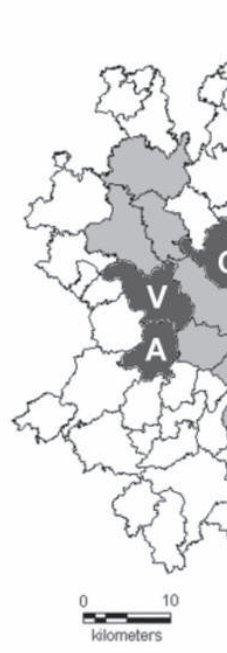

Aire urbaine de Clermont-Ferrand

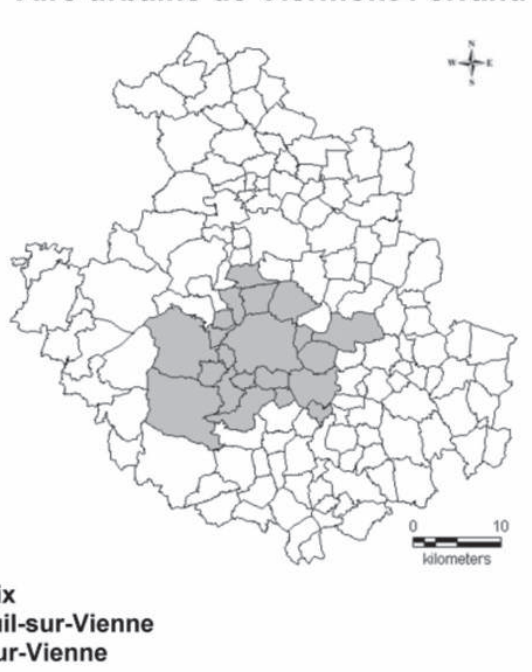

Figure 3 : Les communautés d'agglomération et les aires urbaines, ou l'incohérence des échelles d'élaboration des PDU Agglomeration communities and functional urban regions, or the incoherence of PDU drawing up scales

des politiques mises en ouvre. Une coordination entre les groupements intercommunaux de chaque aire urbaine semblerait plus pertinente, afin d'atténuer les incohérences de certaines structures intercommunales ${ }^{5}$, en rejoignant le concept d'interterritorialité développé par M. Vanier (2008).

\section{Un certain délaissement des enjeux de pollution de l'air}

Il transparaît par ailleurs que le contrôle de la pollution de l'air ne constitue pas le point central des plans de déplacements, alors qu'il détermine à l'origine la mise en œuvre des PDU. L'objectif environnemental se trouve donc écarté des préoccupations des aménageurs, à l'exception de La Rochelle, qui a engagé, dès les années 1970, des actions prenant en considération les impacts de la circulation automobile sur la qualité de vie urbaine.

Même si la pollution n'atteint pas les niveaux enregistrés en région parisienne ou au sein de territoires fortement industrialisés, les problèmes environnementaux existent et les habitants de ces agglomérations intermédiaires pourraient tirer profit du développement de modes de déplacement plus respectueux de la qualité de l'air.

5. Cette perspective pourrait être, dans certains cas, modifiée par une refonte de la carte de l'intercommunalité (issue de la loi du 16 décembre 2010 portant réforme des collectivités territoriales), dont l'un des buts est de rationaliser le périmètre des EPCI existants.
Pourtant, les politiques de ces trois agglomérations se caractérisent par des limites similaires, témoignant d'une prise en compte assez faible de la pollution de l'air. Cette thématique n'apparaît pas capitale dans les prises de décision des trois agglomérations et elle se trouve fortement délaissée face aux enjeux de réchauffement climatique et de développement durable ${ }^{6}$. Ces deux dernières thématiques sont largement dominantes au sein des Agendas 21 locaux et le dioxyde de carbone, principal Gaz à Effet de Serre (GES), est la seule substance évoquée (Glandus, 2010). Notamment, le phénomène d'échelle planétaire que constitue l'effet de serre occupe une place centrale dans les actions de communication actuelles et les politiques environnementales locales s'orientent essentiellement vers la diminution des émissions de dioxyde de carbone, en négligeant de prendre en compte les principaux polluants atmosphériques que sont le dioxyde d'azote, le dioxyde de soufre, le monoxyde de carbone, les particules en suspension, le benzène, l'ozone ou encore le plomb ${ }^{7}$. En conséquence,

6. La notion de développement durable s'est développée au cours des années 1990, après sa définition par le Rapport Brundtland, publié en 1987 par la Commission mondiale sur l'environnement et le développement de l'ONU : «Le développement durable est un développement qui répond aux besoins du présent sans compromettre la capacité des générations futures de répondre aux leurs. »

7. Ces polluants, considérés comme les plus dangereux, doivent être obligatoirement surveillés au moyen de stations fixes dans les agglomérations de plus de 100000 habitants, comme imposé par le décret 98-360 du 6 mai 1998. En outre, la directive 2004/107/CE du 15 décembre 2004 a 
les actions en faveur des réaménagements de voirie, du stationnement et du développement des transports en commun tardent à être mises en œuvre.

Aucune action n'est entreprise pour sensibiliser les populations à la nécessité de modifier leurs habitudes dans un souci de réduction des émissions atmosphériques. Certes, le réchauffement climatique est un problème capital, qui nécessite une attention forte, mais les impacts de la pollution locale sont plus faciles à appréhender, car touchant directement à la qualité de vie et à la santé au niveau local. Il semblerait donc utile que les questions de pollution de l'air et de réchauffement climatique soient prises en compte de manière commune, leurs principales causes étant similaires (Richert, 2007; Global Atmospheric Pollution Forum, 2009; European Federation of Clean Air and Environmental Protection Associations, 2010). Une diminution de l'usage de l'automobile, principal objectif des PDU, s'inscrit dans le sens d'une baisse des émissions polluantes, mais aussi de la consommation énergétique et des émissions de dioxyde de carbone. Néanmoins, certaines actions efficaces contre l'un de ces problèmes peuvent avoir des effets négatifs sur l'autre (Airparif, 2009). Une gestion intégrée et synchronisée semble donc essentielle, autour d'une gouvernance rassemblant tous les acteurs de la société, publics comme privés; cela pouvant permettre à ces deux questions de gagner en crédibilité comme en légitimité et de renforcer la rapidité et l'efficacité de mise en œuvre des actions.

Un pas a été franchi avec la loi du 12 juillet 2010 portant engagement national pour l'environnement, dite Grenelle 2, qui a prévu l'instauration de «Schémas régionaux climat-air-énergie » (SRCAE), visant à atténuer les effets du changement climatique, à prévenir ou réduire la pollution atmosphérique et à fixer les objectifs à atteindre en matière de valorisation du potentiel énergétique terrestre. Cependant, ces objectifs, de même que les thématiques relatives aux transports évoquées dans le Grenelle de l'environnement, sont davantage traités sous l'angle des émissions de gaz à effet de serre - du principal d'entre eux surtout, le dioxyde de carbone - que de la pollution atmosphérique dans son ensemble. De plus, le décret d'application a tardé

élargi la liste des polluants surveillés à d'autres substances, dont quatre métaux (arsenic, cadmium, mercure, nickel et hydrocarbures aromatiques polycycliques). à être publié (18 juin 2011), beaucoup de régions ont pris du retard dans l'élaboration des SRCAE et le cadre restrictif imposé par l'Etat a conduit bon nombre d'entre elles à revoir leurs ambitions à la baisse, a fortiori dans un contexte économique difficile.

Pourtant, au-delà des préoccupations d'optimisation des modes de déplacement, la qualité de l'air peut également jouer un rôle dans le cadre de la promotion touristique et démographique, par le biais de l'image des territoires. En effet, la qualité de l'air, qui est associée à la qualité de vie, «peut constituer un élément marquant, éventuellement incontournable de l'environnement rural ou urbain, contribuant à une caractérisation forte des espaces » (Roussel et Charles, 2007).

Cependant, il semble que les agglomérations ne communiquent que très faiblement sur le thème de la pollution atmosphérique, aussi bien par le biais d'informations relatives aux niveaux de pollution (via l'affichage des indices ATMO, justement créés pour informer le public) que par l'intermédiaire de campagnes de sensibilisation.

\section{Une faible coopération entre acteurs}

En conséquence de ce délaissement des enjeux de pollution de l'air, il ressort un grand détachement des politiques d'aménagement et de transport vis-àvis des principaux facteurs influençant la pollution (comportements des habitants en termes de déplacements, configuration des centres-villes, etc.). Concrètement, il n'existe que peu ou pas de lien entre ces thématiques. La pollution de l'air a longtemps été réduite au problème industriel, ce depuis l'adoption du décret de 1810 sur les établissements dangereux, incommodes et insalubres. Ainsi, la croissance de la pollution liée aux mobilités a été difficilement appréciable pour les différents acteurs, qu'ils soient aménageurs ou citoyens.

D'une part, il semblerait cohérent que les mesures réalisées par les AASQA soient utilisées dans le cadre des politiques d'aménagement. Cela peut se traduire par la demande d'études ciblées de la part des communautés d'agglomération ou municipalités, la prise en compte des résultats dans les réaménagements (restriction de circulation, sens uniques, piétonisation, couloirs de bus, etc.), puis l'analyse des impacts de ceux-ci sur la qualité de l'air. Mais 
la réalité montre que les relations entre associations de surveillance et communautés d'agglomération sont globalement peu intenses. À Limoges comme à Clermont-Ferrand, aucune décision n'a jusqu'à présent été prise en réponse à des mauvais résultats de qualité de l'air. À La Rochelle, l'association a davantage été intégrée aux projets d'aménagement (prise en compte de certains résultats de mesures et demande d'étude relative à l'influence des modifications de circulation sur la qualité de l'air). De plus, si les associations ont été consultées lors de l'élaboration des PDU à La Rochelle et Clermont-Ferrand, cela n'a pas été le cas à Limoges. Ainsi, il semble surprenant que les AASQA travaillent de manière isolée, sans lien avec les communautés d'agglomération qui contribuent pourtant à leur financement, alors que le développement d'une prévention d'ordre structurel pourrait s'avérer nécessaire, dans le but de réduire la pollution urbaine sur le long terme.

D'autre part, la coopération peut également concerner les trois principaux acteurs de la vie urbaine, constituant un triangle de "gouvernance" (Roussel et Charles, 2006) : les pouvoirs publics, les intérêts privés et la société civile. Les entreprises et administrations ont un rôle à jouer auprès de leurs salariés, afin d'encourager les déplacements alternatifs à la voiture, par le biais des Plans de Déplacements d'Entreprise et d'Administration (PDE/PDA). Quelques-uns ont déjà été instaurés mais ils restent encore rares. Quant aux communautés d'agglomération, elles pourraient davantage intégrer les habitants - principaux acteurs des mobilités - à l'élaboration des politiques de transports. La conception des PDU a permis aux citoyens de participer aux discussions. Mais si l'ensemble de la population a été consulté à La Rochelle, seules les associations d'usagers l'ont été à Clermont-Ferrand, alors que les habitants de Limoges ont seulement été informés de la mise en place du PDU avant son élaboration. Même s'il est difficile de connaître le réel poids des habitants dans l'élaboration du document, cette volonté de concertation instaurée à La Rochelle a au moins permis une sensibilisation et une certaine responsabilisation du grand public. Mais de manière générale, il semble que l'intérêt de la population ne soit pas suffisamment éveillé par des actions de communication, d'information et de sensibilisation aux enjeux de la pollution et à la nécessité d'utiliser les modes de transport alternatifs. Du reste, la participation aux enquêtes publiques, menées entre les phases d'élaboration et de validation des PDU, a été relativement faible, et ce au niveau national (Certu et ADEME, 2002). Or, la mise en application des directives nécessite une appropriation par les citoyens et il est difficilement concevable de mener une politique de préservation environnementale sans une large concertation autour de la mobilité automobile (Kergomard, 2007).

Enfin, la coopération peut être établie entre les collectivités locales elles-mêmes, et notamment entre les Autorités Organisatrices des Transports Urbains (AOTU), afin de dépasser les limites d'échelle des réseaux de transports en commun urbains et de mieux coordonner les dessertes et horaires de passage. Les réseaux départemental et régional représentent une petite partie de l'offre générale proposée au sein des communautés d'agglomération. Mais ils ont pourtant la possibilité de proposer une desserte bien plus grande, adaptée aux agglomérations «fonctionnelles », en lien avec les mobilités actuelles. L'objectif serait alors de proposer un vaste réseau de transports en commun et non plusieurs réseaux parallèles, à l'image de ce qui a été introduit en Charente-Maritime avec la constitution d'un syndicat mixte en 1999, regroupant les principales AOTU du département de CharenteMaritime, dans le but de développer l'intermodalité entre les différents réseaux. L'instauration d'un titre de transport unique et une meilleure coordination des horaires constituent des avantages non négligeables pour une plus grande utilisation des modes alternatifs. On peut également considérer qu'une coopération permettrait de dépasser quelque peu les problèmes financiers des collectivités en charge des transports publics, notamment avec le fort désengagement de l'Etat depuis les années 1990 et surtout depuis 2004.

L'information et la sensibilisation du public paraissent donc essentielles, de même qu'une meilleure coordination entre aménagements et pollution de l'air, afin que les actions concrètes répondent à ce qui a été introduit et imposé par la loi sur l'air et la loi SRU et donc que les PDU répondent, comme prévu initialement, aux problèmes de pollution de l'air. La réorganisation urbaine peut, elle aussi, passer par la prise en compte des usages quotidiens des citadins, une concertation, des débats et négo- 
ciations entre tous les acteurs, dès la conception du projet, afin de mettre en cohérence la planification voulue et les intérêts des habitants (Da Cunha et Kaiser, 2009; Zunino, 2009) : la conception d'une nouvelle forme d'organisation des villes doit se vouloir collective, associant professionnels de l'urbanisme et de l'architecture urbaine, communes, intercommunalités et citoyens. Néanmoins, toutes les orientations proposées dans le cadre des plans de déplacements doivent être regardées à l'aune des réalités actuelles, qui posent un certain nombre de limites.

\section{Des actions perfectibles pour une réelle baisse de la pollution urbaine}

Le recours marqué à la voiture pour les déplacements quotidiens (75\% des déplacements domicile-travail en moyenne) témoigne d'un fort ancrage culturel du mode individuel et de lacunes dans le développement des modes alternatifs, et notamment des transports publics. En effet, les actions menées dans le cadre des déplacements urbains témoignent d'un faible engagement global des trois agglomérations.

De façon générale, la volonté de contrôle de l'étalement périurbain, qui apparaît comme un objectif partagé par les PDU, est pour l'instant assez délaissée. L'association, engagée par la loi SRU, entre planification des transports, aménagement urbain et pollution de l'air, n'est pas encore concrètement réalisée. La place accordée à l'automobile reste encore très importante et l'usage du véhicule particulier demeure assez libre, voire renforcé, en espace périurbain, comme en témoigne la réalisation de nouveaux axes de circulation et de pôles d'attraction périphériques à proximité des voies rapides. Les communautés d'agglomération n'ambitionnent pas vraiment de réduire la circulation automobile, mais plutôt de la déplacer des centres-bourgs et du centre-ville des communes-centres vers des axes de contournement. Un schéma dual ressort alors de cette organisation urbaine, entre des centres-villes plutôt orientés vers les transports en commun, les modes doux, le partage de la voirie et la restriction du stationnement, et des espaces périurbains axés sur la primauté de l'automobile (Offner, 2003). Or, la seule réduction du trafic automobile en centreville, même si elle est nécessaire (compte tenu de la vulnérabilité de ces espaces à la pollution), n'est pas suffisante pour espérer une amélioration de la qualité de l'air à l'échelle d'une agglomération et une diminution des niveaux moyens de pollution de fond. Mais actuellement, la présence des voitures reste encore assez marquée en centre-ville, générant encombrements de voirie, nuisances et pollution. Cependant, la question de la restriction du trafic automobile se heurte à des enjeux divergents, qui dépassent le seul cadre de la pollution de l'air : la fréquentation commerciale des centres-villes face à la concurrence des espaces commerciaux et d'activités périphériques. Dans les faits, les avantages de la piétonisation des hypercentres ne fait pas l'objet d'un consensus auprès des commerçants et de nombreux parcs de stationnement sont réalisés pour satisfaire les demandes de ceux-ci, alors que les orientations devraient se traduire par une réduction du nombre total de places.

Par ailleurs, les actions destinées à accroître le recours aux autres modes de transport ne répondent pas, bien souvent, aux volontés exposées dans les PDU. La Rochelle présente, certes, un bon développement de son réseau cyclable (plus de $160 \mathrm{~km}$ ), couplé à une offre de libre-service vélos, sur le modèle de celui de Lyon ou de Paris. Mais à Clermont-Ferrand et surtout à Limoges, les voies réservées aux cyclistes sont peu nombreuses (respectivement 53 et $57 \mathrm{~km}$ ), peu sécurisées et manquent de continuité. De même, les propositions d'intermodalité ressemblent surtout à une multimodalité, car manquant de coordination pour faciliter le passage d'un mode à un autre. Les parcs relais, ayant pour vocation de permettre aux automobilistes de laisser leur véhicule durant plusieurs heures, voire la journée, afin d'emprunter les modes collectifs de transports, pâtissent d'une inégale répartition au sein des agglomérations et d'une localisation parfois peu adaptée; du fait d'une situation trop centrale, certains ne permettent pas d'éviter les encombrements des voies de circulation. De plus, les parcs de Limoges souffrent d'une capacité assez restreinte ( 1,7 places pour 1000 habitants de la communauté d'agglomération, contre respectivement 6,6 et 6 places pour 1000 habitants à La Rochelle et Clermont-Ferrand). Le fonctionnement des parcs relais de Clermont-Ferrand manque également de clarté, l'utilisation de certains n'étant pas couplée avec celle des transports en commun via un tarif 
spécifique et avantageux (pourtant caractéristique, normalement, de ces parcs). Enfin, le succès de ces parcs dépend également de la qualité de l'offre des réseaux de transports en commun, actuellement inégale.

Le développement des transports en commun a jusqu'alors été limité et les réseaux urbains présentent de multiples faiblesses, relevées par les habitants eux-mêmes (Glandus, 2010). L'incohérence des échelles d'application des politiques et le manque de synchronisation entre les réseaux des différentes AOTU (communauté d'agglomération, département, région), préalablement soulignés, entraînent des lacunes dans les dessertes : celles-ci se révèlent assez inégales, privilégiant les espaces denses des agglomérations (communes-centres et certaines communes de première couronne), notamment les centres-villes, et ne correspondent pas aux attentes des habitants. On peut ainsi noter une dégradation de l'offre avec l'éloignement des centres urbains et un manque d'adaptation aux évolutions de l'urbanisation. Les zones d'activités excentrées sont insuffisamment desservies par les lignes de transports en commun et les problèmes concernent notamment les communes périphériques de deuxième couronne qui, si elles sont de plus en plus attractives pour la population, souffrent d'une faible densité d'habitat et d'une répartition anarchique des habitations; ceci révélant les limites de la périurbanisation désordonnée pour l'organisation des réseaux. En effet, le réseau urbain de La Rochelle, plus équilibré entre communes de première et deuxième couronnes, témoigne de l'avantage d'un habitat groupé, par opposition à l'habitat dispersé et le mitage qui caractérisent les agglomérations de Limoges et Clermont-Ferrand. De plus, la qualité de service est restreinte par des fréquences de passage parfois faibles, un développement insuffisant des couloirs réservés aux véhicules de transports en commun - qui restent peu nombreux et trop dispersés - et le manque de confort de certains véhicules (accessibilité parfois difficile, manque de places assises) et de certains arrêts (nombre d'abribus insuffisant). Il résulte donc de tout cela un déficit global de compétitivité des transports en commun face à la voiture.

Enfin, les limites sont inhérentes aux réalités actuelles, aux habitudes des populations et à la place centrale qu'occupe l'automobile dans la société. La tendance générale s'oriente plutôt vers la volonté de posséder un pavillon individuel à l'écart des zones denses et un, voire plusieurs, véhicule particulier, et témoigne ainsi de forts comportements individualistes, à l'opposé de la promotion des modes collectifs. À ce titre, la densification urbaine peut sembler inconfortable, dans un contexte orienté vers la recherche d'espace individuel. Une ville compacte risque en effet de générer une réduction des espaces de vie, pouvant être considérée comme néfaste à la qualité de vie et ne correspondant pas aux attentes des habitants. De ce fait, renoncer, même partiellement, à l'automobile et au confort actuel, reviendrait à changer radicalement de mode de vie et semble difficile, voire impossible à court terme.

En réalité, la mise en œuvre de l'ensemble des mesures proposées dans le cadre des PDU pourrait être souhaitable dans le but de diminuer l'usage de la voiture au quotidien et d'espérer une amélioration de la qualité de l'air plus efficace, puisque jusqu'à présent, les quelques effets constatés n'ont été que très ponctuels, dans le temps comme dans l'espace (organisation de journées sans voitures; restriction de la circulation dans seulement une ou deux rues qui conduit à un report du trafic vers d'autres voies mais pas à une réelle diminution). Mais l'évolution ne pourra nécessairement se faire que très progressivement et il est évident qu'on ne peut bannir totalement l'automobile. Dans ce contexte, la sensibilisation des populations aux enjeux environnementaux prend toute son importance, la contrainte semblant indispensable pour dépasser les réflexes individualistes mais devant aussi s'accompagner de réelles améliorations dans les offres de transports, attendues par une majorité d'habitants (Glandus, 2010).

\section{Conclusion}

En dépit d'une législation commune, les agglomérations s'investissent de façon inégale et ne répondent pas de la même façon à ce qui, normalement, constitue un impératif. Mais il ressort surtout deux fortes similitudes liées, d'une part, à la quasiabsence de prise en compte de la pollution de l'air au profit du réchauffement climatique et, d'autre part, à l'incohérence générale des échelles d'élaboration des PDU. De ces caractères se dégagent des faiblesses qui portent atteinte à l'efficacité des politiques mises en œuvre. Il ne transparaît pas de réelle 
coordination entre les institutions elles-mêmes, à savoir entre communautés d'agglomérations et associations de surveillance. En conséquence il n'existe pas, ou très peu, d'analyses précises quant aux impacts des réaménagements urbains sur les niveaux de pollution, qui permettraient pourtant d'optimiser les actions. Du reste, la question de la pollution de l'air n'est que très rapidement évoquée dans les PDU eux-mêmes et aucun objectif chiffré précis n'est mentionné. Ainsi, la place accordée à la voiture reste encore très grande et les actions de promotion des modes de transports alternatifs ne peuvent permettre d'obtenir des effets marqués sur la qualité atmosphérique urbaine; ce en lien avec un manque d'adaptation des réseaux aux besoins des habitants - en termes de desserte, d'horaires comme de confort - et avec une quasi-absence d'actions d'information et de sensibilisation des populations.

Quoiqu'il en soit, compte tenu des divers enjeux sociétaux et financiers que ces réaménagements soulèvent, ces mesures de planification des transports semblent difficilement réalisables de manière rapide et ne constituent probablement qu'un levier d'importance assez modérée pour limiter les émissions polluantes issues des mobilités automobiles et, de ce fait, les niveaux de pollution en milieu urbain. Les progrès technologiques apportés aux véhicules et aux carburants présentent, a priori, des qualités vraisemblablement plus à même de diminuer les problèmes de pollution, car ne contraignant pas à des modifications majeures des habitudes. Toutefois, ce paramètre ne constituerait pas la panacée au regard de l'étude réalisée par le Commissariat Général au Développement Durable (2010), qui met en avant l'existence d'un « effet rebond ", témoignant d'une compensation partielle des améliorations technologiques (moindre consommation des véhicules permettant un gain financier) par un accroissement de la mobilité. Dans ce contexte, la hausse du prix du carburant serait alors susceptible d'encourager les usagers de la voiture à s'orienter vers d'autres modes, à condition que le développement des offres de transport alternatifs soit efficace et qu'en parallèle, la circulation automobile soit contrainte.

Mais avant tout, la prise de conscience des problèmes de pollution de l'air peut constituer une base utile, si elle est partagée par les différents acteurs, des politiques aux citoyens. Surtout, il paraît légitime que la pollution de l'air reprenne sa place dans les préoccupations des agglomérations intermédiaires, alors que l'effet de serre focalise aujourd'hui toute l'attention. La société civile pourrait alors occuper une place centrale dans cette lutte contre les émissions polluantes, le succès des politiques de déplacement dépendant du degré de volonté et d'acceptation des nouvelles orientations par les populations.

\section{Bibliographie}

Airparif, 2009. Gaz à effet de serre et polluants atmosphériques - Vrais liens et fausses idées, Airparif Actualité, $\mathrm{n}^{\circ} 33$, p. 2-3.

Bartholomew K., Ewing R., 2009. Land use-transportation scenarios and future vehicle travel and land consumption: a meta-analysis, Journal of the American Planning Association, vol. 75, p. 13-27.

Boutefeu E., 2007. Végétaliser les villes pour atténuer les îlots de chaleur urbains, Techni-Cités, n 129, p. 20-21.

Burton E., 2000. The compact City: just or just compact? A preliminary analysis, Urban Studies, vol. 37, p. 1969-2006.

Certu, ADEME, 2002. Bilan des PDU de 1996 à 2001, Certu Ministère de l'Equipement, des Transports et du Logement, Lyon, 369 p.

Cire (Cellule Interrégionale d’épidémiologie) RhôneAlpes Auvergne, 2005. Impact sanitaire de la pollution atmosphérique sur l'agglomération de Clermont-Ferrand, $44 \mathrm{p}$.

CITEPA, 2011. Emissions dans l'air en France métropolitaine, http://www.citepa.org.

Colombert M., 2008. Contribution à l'analyse de la prise en compte du climat urbain dans les différents moyens d'intervention sur la ville, Thèse de doctorat, Université Paris-Est, $538 \mathrm{p}$.

Commissariat Général au Développement Durable, 2010. CO2 et activités économiques de la France. Tendances 19902007 et facteurs d'évolution, 52 p.

Daв W., Roussel I., 2001. L'air et la ville, Hachette Littératures, Paris, $219 \mathrm{p}$.

Da Cunha A., Kaiser C., 2009. Densité, centralité et qualité urbaine : la notion d'intensité, outil pour une gestion adaptative des formes urbaines?, Urbia-Les cahiers du développement urbain durable, $\mathrm{n}^{\circ}$ 9, p. 13-56.

DAHECH S., 2007. Le vent à Sfax (Tunisie), impacts sur le climat et la pollution atmosphérique, Thèse de doctorat, Université Paris 7, $309 \mathrm{p}$.

DATAR, 2010. Villes intermédiaires et leurs espaces de proximité, Groupe de prospective Territoires 2040, document de travail.

De Ridder K., Lefebre F., Adriaensen S., Arnold U., Beckroege W., Bronner C., Weber C., 2008. Simulating the impact of urban sprawl on air quality and population exposure in the German Ruhr area. Part II: Development 
and evaluation of an urban growth scenario, Atmospheric Environment, vol. 42, p. 7070-7077.

D'Helf-Blanchard M., 2005. Synthèse des évaluations d'impact sanitaire de la pollution atmosphérique urbaine en France de 1995 à 2002, Bulletin Épidémiologique hebdomadaire, $\mathrm{n}^{\circ} 19$, p. 85-86.

Dockery D., Pope A., 1996. Epidemiology of acute health effects: summary of time-series studies, dans WiLson R. (dir.), Particles in our Air: concentrations and health effects, Boston, Harvard University Press, p. 123-147.

Drobenko B., 2002. Les villes durables, dans Mondialisation et

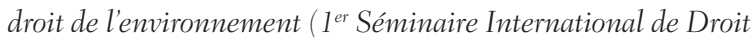
de l'Environnement: Rio +10), Rio de Janeiro, Université de Limoges, CIDCE, ESMPU, p. 149-171.

Dupuy G., 1995. Les territoires de l'automobile, Economica, Paris, $216 \mathrm{p}$.

Emangard P. H., 1994. Espace urbain et efficacité des réseaux de province, Transports urbains, $\mathrm{n}^{\circ} 83$, p. 5-16.

European Federation of Clean Air and Environmental Protection Associations, 2010. Linking air pollution and climate change, http://efca.net/.

Frank L. D., Sallis J. F., Conway T. L., Chapman J. E., SaeLEns B.E., Bachman W., 2006. Many pathways from land use to health: associations between neighborhood walkability and active transportation, body mass index, and air quality, Journal of the American Planning Association, vol. 72, p. 75-87.

Glandus L.-M., 2010. Géographie de l'air et politiques urbaines - L'exemple de villes moyennes de l'ouest français: La Rochelle, Limoges et Clermont-Ferrand, Thèse de doctorat, Université de Limoges, 424 p.

Glandus L.-M., Dahech S., Beltrando G., 2011 . Variabilité spatio-temporelle du CO dans le centre de Limoges : apport des mesures itinérantes, Pollution atmosphérique, $\mathrm{n}^{\circ} 210$, p. $217-224$.

Global Atmospheric Pollution Forum, 2009. Main Conclusions, dans Conference on Air pollution and climate change. Developing framework for integrated co-benefits strategies, Stockholm, Stockholm Environment Institute (SEI), [www. gapforum.org].

Hixson M., Mahmud A., Hu J., Bai S., Niemeier D. A., Handy S. L., Gao S., Lund J. R., Sullivan D. C., Kleeman M. J., 2010. Influence of regional development policies and clean technology adoption on future air pollution exposure, Atmospheric Environment, vol. 44, p. 552-562.

Kaufmann V., Jemelin C., Guidez J.-M., 2001. Automobile et modes de vie urbains : quel degré de liberté?, La Documentation française - Predit, Paris, 167 p.

Kenworthy J. R., Laube F. B., 1999. Patterns of automobile dependence in cities: an international overview of key physical and economic dimensions with some implications for urban policy, Transportation Research Part A, vol. 33, p. 691-723.

Kergomard C., 2007. Introduction, dans Scarwell H. J., Kergomard C., Laganier R. (dir.), Environnement et gouvernance des territoires : enjeux, expériences et perspectives en région Nord-Pas-de-Calais, Villeneuve-d'Ascq, Presses Universitaires du Septentrion, p. 21-30.
LE Roux S., 2008. Energie et développement urbain durable. Analyse des stratégies locales et essai de prospective pour les villes moyennes européennes, Thèse de doctorat, Université de Limoges, 420 p.

Lévy J., Lussault M., 2003. Dictionnaire de la géographie et de l'espace des sociétés, Belin, Paris, 1033 p.

Lonati G., Giugliano M., Cernuschi S., 2006. The role of traffic emissions from weekends' and weekdays' fine PM data in Milan, Atmospheric Environment, n 40, p. 59986011.

Mainet H., Edouard J.-C., 2009. Les limites de l'aire urbaine : la difficile cohérence entre espaces fonctionnels et territoires de gestion, dans Chignier-Riboulon F., ClermontFerrand, ville paradoxale, Clermont-Ferrand, Presses Universitaires Blaise Pascal, p. 13-15.

Marshall J. D., McKone T. E., Deakin E., Nazaroff W. W., 2005. Inhalation of motor vehicle emissions: effects of urban population and land area, Atmospheric Environment, vol. 39, p. 283-295.

Masclet P., 2005. Pollution atmosphérique - Causes, conséquences, solutions, perspectives, Ellipses, Paris, 213 p.

Nazelle, A. de, Rodriguez D. A., Crawford-Brown D., 2009. The built environment and health : impacts of pedestrian-friendly designs on air pollution exposure, Science of the Total Environment, vol. 407, p. 2525-2535.

Newman P. W. G., Kenworthy J. R., 1989. Cities and automobile dependence: an international sourcebook, Gower Technical, Aldershot, 388 p.

Newman P. W. G., Kenworthy J. R., 1999. Sustainability and cities - Overcoming automobile dependence, Island Press, Washington D.C., 450 p.

Offaner J.-M., 2003. Les Plans de Déplacements Urbains (1996-2002), dans Pumain D., Mattei M.-F. (dir.), Données urbaines, Paris, Anthropos-Economica, p. 379-386.

Paquot T., 2009. Vers un urbanisme sensoriel, Propos recueillis par Sophie Chapelle, [www.mowvements.info/Vers-un-urbanisme-sensoriel.html].

Pouyanne G., 2004. Des avantages comparatifs de la ville compacte à l'interaction forme urbaine-mobilité. Méthodologie et premiers résultats, Les cahiers scientifiques $d u$ transport, $\mathrm{n}^{\circ} 45$, p. 49-82.

Richert P., 2007. Qualité de l'air et changement climatique : un même défi, une même urgence, La Documentation française, Paris, 143 p.

Roussel I., Charles L., 2006. Pollution atmosphérique et proximité, Pollution atmosphérique, n 190, p. 175-182.

Roussel I., Charles L., 2007. L'impossible territorialisation de la qualité de l'air, dans Scarwell H. J., Kergomard C., LAGANIER R. (dir.), Environnement et gouvernance des territoires : enjeux, expériences et perspectives en région NordPas-de-Calais, Villeneuve-d'Ascq, Presses Universitaires du Septentrion, p. 109-164.

Schwertzer L., Zhou J., 2010. Neighborhood air quality, respiratory health and vulnerable populations in compact and sprawled regions, Journal of the American Planning Association, vol. 76, p. 363-371. 
VANIER M., 2008. Le pouvoir des territoires. Essai sur l'interterritorialité, Anthropos-Economica, Paris, 160 p.

Vhassopoulou C.A., 1999. La lutte contre la pollution atmosphérique urbaine en France et en Grèce. Définition des problèmes publics et changement de politique, Thèse de doctorat, Université Paris 2, 640 p.

WiEL M., 2002. Ville et automobile, Descartes \& Cie, Paris, $140 \mathrm{p}$.
Xiaomin X., Zhen H., Jiasong W., 2006. The impact of urban street layout on local atmospheric environment, Building and Environment, $\mathrm{n}^{\circ} 41$, p. 1352-1363.

Zunino G., 2009. Plaidoyer pour une ville intense, Urbia-Les cahiers du développement urbain durable, $\mathrm{n}^{\circ}$ 9, p. 57-76. 